\title{
Calculation of the surface tension of liquid copper from atomistic
}

\section{Monte Carlo simulations.}

Emeric Bourasseau ${ }^{1}$, Ahmed-Amine Homman $^{1}$, Olivier Durand ${ }^{1}$, Aziz Ghoufi $^{2}$, and Patrice Malfreyt ${ }^{3}$

${ }^{1}$ CEA / DAM / DIF, F-91297 Arpajon Cedex, France

${ }^{2}$ Institut de Physique de Rennes, UMR CNRS 6251, Université Rennes 1, 263 avenue du Général Leclerc, 35042 Rennes, France

${ }^{3}$ Institut de Chimie de Clermont-Ferrand, ICCF, UMR CNRS 6296, BP 1048, F-63000, Clermont-Ferrand

Received: June 5, 2013/ Revised version: date

\begin{abstract}
Monte Carlo simulations of heterogeneous systems of copper at liquid-vapor equilibrium have been performed at several temperatures from 1400 to $2000 \mathrm{~K}$, using the EAM potential of Zhou et al. (Phys. Rev. B 69, 144113 (2004)). Surface tension of the corresponding planar interfaces has been evaluated using thermodynamic and mechanical approaches. We have investigated the impact of the potential and the temperature on the surface tension of liquid copper. For the first time, calculation results are in very good agreement with experiments with a maximum deviation of $2 \%$ from experiments. Additionally, the Monte Carlo simulations provide a temperature coefficient (the derivative of surface tension in regard with temperature) in excellent agreement with the experimental coefficient. This was one of the main challenges of the present simulations.
\end{abstract}

PACS. PACS-key discribing text of that key - PACS-key discribing text of that key

\section{Introduction}

Surface tension of liquid metals is an important parameter of many materials design. This property impacts on the ability of a liquid to wet a solid surface, and wetting phenomena appear to be of great importance in many prepa-

Correspondence to: emeric.bourasseau@cea.fr ration methods. Unfortunately, measurements of surface tension are still difficult, whereas many methods have been proposed, mainly because it is not easy to determine the influence of impurities present in the melt. It results that the temperature dependence of the surface tension of liquid metals is not well-known from experimental viewpoint. It has been established recently $[1,2]$ that the accuracy of 
the determination of this property could be very poor and can remain unknown for some metals [2].

As a consequence, a number of empirical models have been developed to predict the surface tension of melts $[2$, 3]. These models are based on correlations between surface and bulk thermodynamic properties and use experimental thermodynamic properties as input. One alternative consists of using the two-phase molecular simulation methods to provide surface tension values. However, these simulations are impacted by a certain number of factors such as potential, surface tension definition and the temperature transferability of the potential. As far as liquid metals are concerned, very few works show calculations of surface tension using atomistic models. Additionally, the simulated surface tensions exhibit significant deviations from experiments [4-6]. As far as we know, only three papers report the calculation of the surface tension of copper by microscopic simulations $[4-6]$. These simulations lead to surface tension values that are underestimated by $20-60 \%$ from experiments. Does it come from the choice of the potential model ? Does the method of calculation impact on the value of the surface tension?

Actually, the calculation of the surface tension of a two-phase system is now robust even though a certain number of factors such as the finite size effects [7-10], the range of interactions [11-14], the truncation effects [11,1517], the mechanical and thermodynamic definitions of the surface tension $[16,18-20]$ and the long range corrections to the surface tension $[13,15,16,20-22]$ can impact the calculated results for this property. Once the methodology was established, molecular simulations of the liquid-vapor interface showed a good reproduction of the temperature dependence of the surface tension for linear and branched alkanes $[13,15,16,23,24]$, cyclic and aromatic hydrocarbons [25-27], ethers [28], water [19,29,30], acid gases [20, 29,31-33], incondensable gases [33] and alcohols [34,35]. The surface tension of more complex interfacial systems such as binary systems [36-38] have also been reproduced by atomistic simulations in large pressure range.

It means that the calculation of the surface tension is now mature on condition that the different parameters (potential, size effects, surface tension definition) that can impact on the results are well-controlled. We propose here to extend the methodologies used for the liquid-vapor interfaces of organic molecules to liquid-vapor interfaces of metals. We aim to reproduce the surface tension of the liquid copper metal at different temperatures and to extract from our simulations the temperature coefficient. The surface tension is calculated using the thermodynamic and mechanical definitions and profiles of the difference between normal and tangential components of the pressure tensor are calculated in the liquid metal. The potential dependence of the surface tension is illustrated through different versions of the embedded atom model (EAM) originally developed by Daw and Baskes [39]. 
Section 2 describes in detail the version of the EAM potential that gives the best reproduction of the temperature dependence of the surface tension of the liquid copper. The different definitions of the surface tension are also presented in Section 2. Section 3 starts with the ability of the different force fields to reproduce the surface tension of the liquid-vapor interface of copper at a given temperature. We finish this section by calculating the surface tension of a large range of temperatures and extracting the temperature coefficient. We conclude in Section 4 by the main results of this paper.

\section{Computational procedures}

\subsection{EAM force field}

Different force fields are available in the literature to describe solid and liquid phases of copper: simple LennardJones potentials (Agrawal et al. [40], Hirschfelder et al. [41]), or more complex EAM potentials (Zhou et al. [42], Belonoshko et al. [43], Cleri et al. [44], Sutton et al. [45]). The usual form of the potential energy of a system composed of $N$ atoms which interact through a EAM force field is given by:

$$
E_{P}=\sum_{i=1}^{N} \sum_{j=1, j \neq i}^{N} \frac{1}{2} \phi\left(r_{i j}\right)+\sum_{i=1}^{N} F\left(\rho_{i}\right)
$$

where $\rho_{i}$ is the atomic density around the atom $i$ :

$$
\rho_{i}=\sum_{j=1, j \neq i}^{N} f\left(r_{i j}\right)
$$

The definitions of $\phi\left(r_{i j}\right), F\left(\rho_{i}\right)$ and $f\left(r_{i j}\right)$ vary depending on which EAM version is used. Following the one proposed by Zhou et al. [42] we used:

$\phi(r)=A \frac{\exp \left(-\alpha\left(\frac{r}{r_{e}}-1\right)\right)}{1+\left(\frac{r}{r_{e}}-\kappa\right)^{20}}-B \frac{\exp \left(-\beta\left(\frac{r}{r_{e}}-1\right)\right)}{1+\left(\frac{r}{r_{e}}-\lambda\right)^{20}}-\phi_{\text {cutoff }}$,

where $\phi_{\text {cutoff }}$ is the value of $\phi(r)$ obtained for $r=$ $r_{\text {cutoff }}$, distance beyond which the interaction between two atoms is neglected. The $f$ function is:

$$
f(r)=f_{e} \frac{\exp \left(-\beta\left(\frac{r}{r_{e}}-1\right)\right)}{1+\left(\frac{r}{r_{e}}-\lambda\right)^{20}}-f_{\text {cutoff }}
$$

where $f_{\text {cutoff } f}$ is the value for $r=r_{\text {cutoff }}$.

Finally, the $F$ function is:

$$
F(\rho)=\left\{\begin{array}{l}
\sum_{i=0}^{3} F_{n i}\left(\frac{\rho}{\rho_{n}}-1\right)^{i}, \rho<\rho_{n}, \rho_{n}=0.85 \rho_{e} \\
\sum_{i=0}^{3} F_{i}\left(\frac{\rho}{\rho_{e}}-1\right)^{i}, \rho_{n} \leq \rho<\rho_{0}, \rho_{0}=1.15 \rho_{e} \\
F_{e}\left[1-\log \left(\left(\frac{\rho}{\rho_{s}}\right)^{\eta}\right)\right]\left(\frac{\rho}{\rho_{s}}\right)^{\eta}, \rho_{0} \leq \rho .
\end{array}\right.
$$

$$
A, B, \alpha, \beta, \kappa, \lambda, r_{e}, f_{e}, F_{n 0}, F_{n 1}, F_{n 2}, F_{n 3}, F_{0}, F_{1}, F_{2} \text {, }
$$

$F_{3}, \rho_{e}, \rho_{s}$ and $\eta$ are parameters taken from the original paper [42].

Since the derivative of the potential is required to calculate the surface tension within the Irving-Kirkwood method, its operational form is

$$
\frac{d U}{d r_{i j}}=\sum_{i} \sum_{j \neq i} \phi^{\prime}\left(r_{i j}\right)+f^{\prime}\left(r_{i j}\right)\left(F^{\prime}\left(\rho_{i}\right)+F^{\prime}\left(\rho_{j}\right)\right),
$$

with 


$$
\left\{\begin{aligned}
\phi^{\prime}\left(r_{i j}\right)= & A\left(-\frac{\alpha}{r_{e}}-\frac{20}{r_{e}} \frac{\left(\frac{r}{r_{e}}-\kappa\right)^{19}}{1+\left(\frac{r}{r_{e}}-\kappa\right)^{20}}\right) \\
& \frac{\exp \left(-\alpha\left(\frac{r}{r_{e}}-1\right)\right)}{1+\left(\frac{r}{r_{e}}-\kappa\right)^{20}} \\
& -B\left(-\frac{\beta}{r_{e}}-\frac{20}{r_{e}} \frac{\left(\frac{r}{r_{e}}-\lambda\right)^{19}}{1+\left(\frac{r}{r_{e}}-\lambda\right)^{20}}\right) \\
& \frac{\exp \left(-\beta\left(\frac{r}{r_{e}}-1\right)\right)}{1+\left(\frac{r}{r_{e}}-\lambda\right)^{20}} \\
f^{\prime}\left(r_{i j}\right)= & f_{e}\left(-\frac{\beta}{r_{e}}-\frac{20}{r_{e}} \frac{\left(\frac{r}{r_{e}}-\lambda\right)^{19}}{1+\left(\frac{r}{r_{e}}-\lambda\right)^{20}}\right) . \\
& \frac{\exp \left(-\beta\left(\frac{r}{r_{e}}-1\right)\right)}{1+\left(\frac{r}{r_{e}}-\lambda\right)^{20}}
\end{aligned}\right.
$$

and the derivative of the density function $F$ is

$$
F^{\prime}(\rho)=\left\{\begin{array}{l}
\sum_{i=1}^{3} i \frac{F_{n i}}{\rho_{n}}\left(\frac{\rho}{\rho_{n}}-1\right)^{i-1}, \rho<\rho_{n}, \rho_{n}=0.85 \rho_{e} \\
\sum_{i=1}^{3} i \frac{F_{i}}{\rho_{e}}\left(\frac{\rho}{\rho_{e}}-1\right)^{i-1}, \rho_{n} \leq \rho<\rho_{0}, \rho_{0}=1.15 \rho_{e} \\
F_{e}\left[\left(1-\frac{\eta}{\rho}\right)\left(\frac{\rho}{\rho_{s}}\right)^{\eta}+(1-\right. \\
\left.\left.\log \left(\left(\frac{\rho}{\rho_{s}}\right)^{\eta}\right)\right)\left(\frac{\eta}{\rho_{s}}\right)\left(\frac{\rho}{\rho_{s}}\right)^{\eta-1}\right], \rho_{0} \leq \rho
\end{array}\right.
$$

\subsection{Surface tension calculation}

The most commonly used methods $[18,46-50]$ for the surface tension calculation are based upon the mechanical route definition and use the tensorial components of the pressure. The definition of Irving and Kirkwood $[48]\left(\gamma_{\mathrm{IK}}\right)$ is based upon the notion of the force across a unit area and takes advantage of expressing the local components of the pressure tensor along the direction normal to the surface. A novel method based upon the thermodynamic definition of the surface tension $\left(\gamma_{\mathrm{TA}}\right)$ has been recently established by Gloor et al. [18] and consists in perturbing the cross-sectional area of the system containing the interface.

\subsubsection{Irving Kirkwood (IK) definition.}

The method of Irving and Kirkwood (IK) [48] expresses the surface tension from the local components of the pressure tensor

$$
\gamma_{\mathrm{IK}}=\frac{1}{2} \int_{-L_{z} / 2}^{L_{z} / 2}\left(p_{\mathrm{N}}\left(z_{k}\right)-p_{\mathrm{T}}\left(z_{k}\right)\right) d z
$$

where $p_{\mathrm{N}}\left(z_{k}\right)$ and $p_{\mathrm{T}}\left(z_{k}\right)$ are the normal and tangential components of the pressure tensor along the normal to the surface, respectively. The method of Irving and Kirkwood [48] (IK) is based upon the notion of the force across a unit area. The pressure tensor is then written as a sum of a kinetic term and a configurational term resulting from the intermolecular forces. Whereas the first term is well defined, the potential term is subjected to arbitrariness because there is no unique way to determine which intermolecular forces contribute to the stress across $d A$. There are many ways of choosing the contour joining two interacting particles. Irving and Kirkwood [48] have chosen the straight line between the two particles. Other choices are possible and results from the lack of uniqueness in the definition of the microscopic stress tensor. The components of the pressure tensor $[46,49,50]$ in the Irving and Kirkwood definition are expressed by

$$
\begin{aligned}
p_{\alpha \beta}\left(z_{k}\right)= & \left\langle\rho\left(z_{k}\right)\right\rangle k_{B} T \mathbf{I}+ \\
& \frac{1}{A}\left\langle\sum_{i=1}^{N-1} \sum_{j=i+1}^{N}\left(\mathbf{r}_{\mathbf{i j}}\right)_{\alpha}\left(\mathbf{F}_{\mathbf{i j}}\right)_{\beta} .\right. \\
& \left.\frac{1}{\left|z_{i j}\right|} \theta\left(\frac{z_{k}-z_{i}}{z_{i j}}\right) \theta\left(\frac{z_{j}-z_{k}}{z_{i j}}\right)\right\rangle
\end{aligned}
$$

where $\mathbf{I}$ is the unit tensor and $T$ is the input temperature. $\alpha$ and $\beta$ represent $x, y$ or $z$ directions. $\theta(x)$ is the 
unit step function defined by $\theta(x)=0$ when $x<0$ and $\theta(x)=1$ when $x \geq 0 . A$ is the surface area normal to the $z$ axis. The distance $z_{i j}$ between two atoms is divided into $N_{s}$ slabs of thickness $\delta z$. Following Irving and Kirkwood, the molecules $i$ and $j$ give a local contribution to the pressure tensor in a given slab if the line joining the atoms $i$ and $j$ crosses, starts or finishes in the slab. Each slab has $1 / N_{s}$ of the total contribution from the $i-j$ interaction. The normal component $p_{N}\left(z_{k}\right)$ is equal to $p_{z z}\left(z_{k}\right)$ whereas the tangential component is given by $\frac{1}{2}\left(p_{x x}\left(z_{k}\right)+p_{y y}\left(z_{k}\right)\right)$. $\mathbf{F}_{\mathbf{i j}}$ is the force between atoms $i$ and $j$ and is expressed as

$$
\mathbf{F}_{\mathbf{i j}}=-\frac{\mathbf{r}_{\mathbf{i j}}}{r_{i j}} \frac{d u\left(r_{i j}\right)}{d r_{i j}}
$$

where the derivative of the potential with respect to the distance is calculated using Eq. (3).

\subsubsection{Test Area}

The second method, called the Test Area Method (TA), has been recently proposed by Gloor et al. [18]. This method comes from a thermodynamic approach, which defines the surface tension $\gamma$ as the work needed to modify the surface of the interface at constant volume. This work can be defined as the free energy variation $d F$ corresponding to the surface variation $d A$ :

$$
d F=\gamma d A-P d V-S d T
$$

and:

$$
\gamma=\left(\frac{\partial F}{\partial A}\right)_{N V T}
$$

where $A$ is the area of the interface, $P$ the pressure, $V$ the volume, $S$ the entropy, $T$ the temperature. The surface tension can thus be calculated by evaluating the free energy $\mathrm{F}$ in the canonical ensemble:

$$
F=-k_{B} T \ln Q_{N V T}
$$

where $Q_{N V T}$ is the canonical partition function:

$$
Q_{N V T}=\frac{V^{N}}{N ! \Lambda^{3 N}} \int_{V} \exp \left(-\frac{U\left(\mathbf{r}^{N}\right)}{k_{B} T}\right) d \mathbf{r}^{N}
$$

where $\Lambda$ is the de Broglie wavelength, $\mathrm{U}$ is the configurational energy, $\mathbf{r}$ is the position vector. The thermodynamic definition expresses the surface tension as:

$$
\gamma=\left(\frac{\partial F}{\partial A}\right)_{N V T}=\lim _{\Delta A \rightarrow 0} \frac{F^{1}-F^{0}}{\Delta A}=\frac{\Delta F}{\Delta A}
$$

$\gamma$ is then calculated from a perturbation of the interfacial area using the free energy perturbation formalism. Two states are defined: (i) a reference state (0) with an area $A_{0}$ and $\mathrm{d} \mathbf{r}_{0}^{N}$ as an infinitesimal volume and (ii) a perturbed state (1) with $A_{1}=A_{0}+\Delta A$ and $\mathrm{d} \mathbf{r}_{1}^{N}$. The perturbation of the interfacial area is applied by modifying the coordinates and the box dimensions from an anisotropic change (with amplitude $\xi$ ): in the case where the heterogeneity takes place in the $\mathrm{z}$ direction, changes are defined as $\eta_{\alpha}^{1}=\eta_{\alpha}^{0}(1 \pm \xi)^{\frac{1}{2}}$, where $\eta$ represents the coordinates of the atoms, with $\alpha=(\mathrm{x}, \mathrm{y})$, and $\eta_{\alpha}^{1}=\eta_{\alpha}^{0}(1 \pm \xi)^{-1}$, with $\alpha$ $=\mathrm{z}$. Thus, the surface tension is expressed as: 


$$
\gamma_{T A}=-\frac{k_{B} T}{\Delta A} \ln \left\langle\exp -\left(\frac{U\left(\mathbf{r}_{1}^{N}\right)-U\left(\mathbf{r}_{0}^{N}\right)}{k_{B} T}\right)\right\rangle_{0}
$$

where $<. .>_{0}$ refers to the canonical average over the reference state, and $U\left(\mathbf{r}_{0}^{N}\right)$ and $U\left(\mathbf{r}_{1}^{N}\right)$ are the energies in the reference and perturbed states, respectively. It is also possible to derive a local expression of $\gamma[51]$ :

$\gamma_{T A}\left(z_{k}\right)=-\frac{k_{B} T}{\Delta A} \ln \left\langle\exp -\left(\frac{U_{z_{k}}\left(\mathbf{r}_{1}^{N_{z_{k}}}\right)-U_{z_{k}}\left(\mathbf{r}_{0}^{N_{z_{k}}}\right)}{k_{B} T}\right)\right\rangle_{0}$

Note that it is also possible to derive a non-exponentional version called TA2 [51]. Following the strategy of thermodynamic integration, $\gamma$ is defined as:

$$
\gamma=\left(\frac{\partial F}{\partial A}\right)_{N V T}=-\frac{k_{B} T}{Q_{N V T}} \frac{\partial Q_{N V T}}{\partial A}
$$

By deriving the expression of $Q_{N V T}$ with respect to A, we obtain (see Ref. [51] for details):

$$
\gamma=\left\langle\frac{\partial U\left(\mathbf{r}_{N}\right)}{\partial A}\right\rangle_{0}
$$

This partial derivative can be calculated explicitly to give the Kirkwood-Buff definition. It is also possible to use finite difference to calculate the surface tension leading to the TA2 working expression:

$$
\gamma_{T A 2}=\left\langle\frac{U\left(\mathbf{r}_{1}^{N}\right)-U\left(\mathbf{r}_{0}^{N}\right)}{\partial A}\right\rangle_{0}
$$

and a local corresponding working expression for TA2

$$
\gamma_{T A 2}\left(z_{k}\right)=\left\langle\frac{U_{z_{k}}\left(\mathbf{r}_{1}^{N_{z_{k}}}\right)-U_{z_{k}}\left(\mathbf{r}_{0}^{N_{z_{k}}}\right)}{\partial A}\right\rangle_{0}
$$

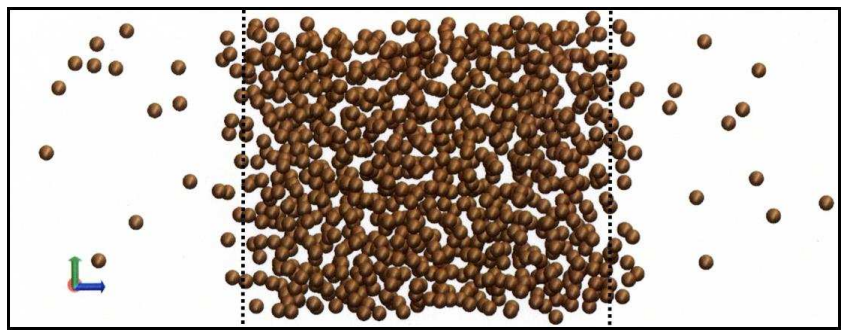

Fig. 1. Configuration of the liquid-vapor interface of copper. The liquid phase is surrounded by two vapour phases and the $z$-direction is perpendicular to the interface.

\section{Results}

\subsection{Convergence of the surface tension}

The starting NVT liquid-vapor configurations were built from NPT configurations. The resulting NPT configurations were modified by increasing the simulation length in the $z$-direction, keeping the liquid phase in the middle of the box (see Figure 1). The periodic boundary conditions were applied in the three directions. MC NVT simulations were performed using standard translation moves. The amplitude of translations was adjusted to give $40 \%$ of accepted moves at the end of the simulations. Surface tension was calculated every 25 Monte Carlo cycles, using the mechanical definition (Eq. 5) and the two versions of the test-area method [18] represented by Eqs. (13) and (17) respectively.

Figure 2 shows the evolution of the surface tension calculated using the three methods (IK, TA and TA2) as a function of the number of cycles at a given temperature. First of all, it appears that the calculation of the surface tension requires approximately $8.10^{5}$ Monte Carlo cycles to converge. As expected from the statistical me- 
chanics, the mechanical definition using the derivative of the energy with respect to the separation distance and the thermodynamic definition using the energy give similar results within $1 \mathrm{mN} \cdot \mathrm{m}^{-1}$. The difference between the different routes is less than the statistical fluctuations. This also means that the EAM potential for which the force and energy expressions are continuous at the cutoff radius allows an identical calculation of the surface tension from the thermodynamic and mechanical definitions. It results that the calculation of the surface tension from Monte Carlo (MC) and molecular dynamics (MD) simulations should be the same with the EAM model. This opens the way of consistent comparisons between the different force fields used either in MC or MD. This was not the case for truncated Lennard-Jones potentials that present discontinuities at the cutoff radius. By using truncated potentials, the calculation of the surface tension is dependent on the way the potentials are truncated and on the method (MC or MD) used to generate the two-phase configurations [11, $15]$.

Figure 3 compares the profile of $\gamma(z)$ along the the $z$-direction calculated using the thermodynamic (Eq. 14) and mechanical (Eq. 5) routes. This local surface tension represents an interesting property to check the stability of the two interfaces. The profiles of the integral of $\gamma(z)$ are shown in Figure 3. We also check that the values of surface tensions calculated from the integration of the profile of $\gamma(z)$ using TA is similar to that calculated from the scalar expression given by Eq. (13) (see the right-hand side graph of figure 3 ). This confirms the decomposition of the surface tension into local elements. From the local elements of the surface tension, we can check that the twophase system presents a fully developed liquid that do not contribute to the surface tension as demonstrated by the plateau in the integration profile. The profiles show two identical positive peaks at the interface regions and two small negative peaks on the gas side of the surface. The difference between the local elements calculated using IK and TA find their origin in the way of distributing the energy into the slabs. However, the integrated value does not depend on the definition used. We can conclude from these profiles that the two-phase simulations of the liquid copper exhibit local profiles expected for mechanical equilibrium of planar interfaces. As far as we know, such profiles have never been shown for the simulations of the liquid-vapor of metals using EAM models.

\subsection{Potential dependence of the surface tension}

Now the methodology of the surface is well-established, we check the performance of the different versions of the EAM potential to predict the surface tension of the liquidvapor surface tension of copper at a given temperature. We compare the results of surface tension using the EAM potentials developed by Zhou et al. [42], Belonoshko et al. [43], Cleri et al. [44] and Sutton et al. [45].

This comparison between the different EAM models is very interesting. The surface tensions are calculated using the two versions of the test-area approach and are represented in Table 1 for a direct comparison with experiments. The TA and TA2 approaches give identical results 


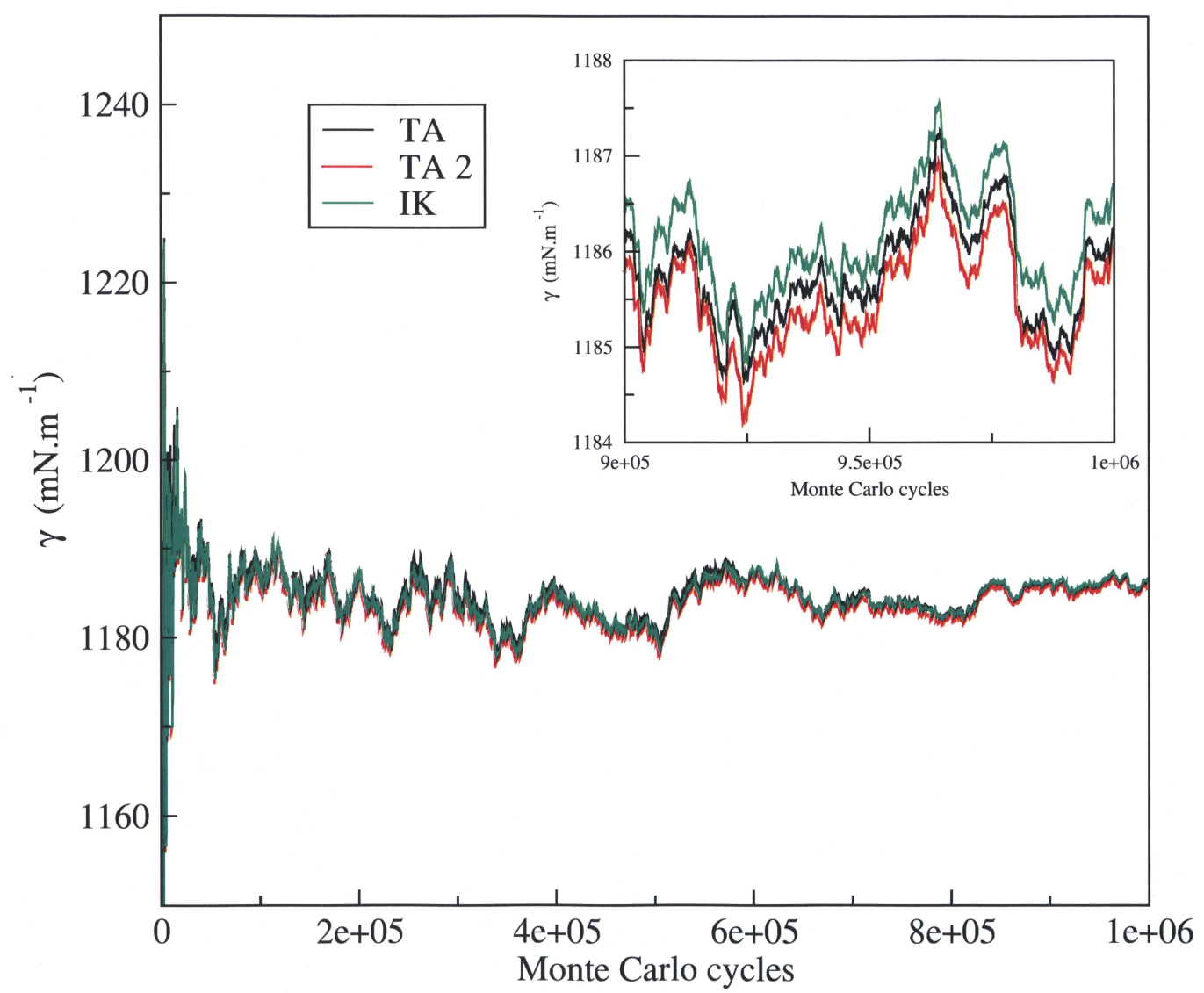

Fig. 2. Evolution of the surface tension calculated using three different methods: Irving-Kirkwood (IK), test-area with the perturbation theory approach (TA) and test-area with the thermodynamic integration approach (TA2) as a function of the number of MC cycles.

Table 1. Surface tension values $\left(\mathrm{mN} . \mathrm{m}^{-1}\right)$ calculated at 1700 K from two versions of the test area method with various EAM potentials. The experimental value is given for comparison.

\begin{tabular}{lcc}
\hline EAM potential & $\gamma_{T A}$ & $\gamma_{T A 2}$ \\
\hline Cleri et al. (Ref. [44]) & $539_{20}$ & $537_{20}$ \\
Sutton et al. (Ref. [45]) & $493_{5}$ & $492_{5}$ \\
Belonoshko et al. - Version 1 - (Ref. [43]) & $413_{12}$ & $413_{12}$ \\
Belonoshko et al. - Version 2 - (Ref. [43]) & $866_{11}$ & $866_{11}$ \\
Zhou et al. (Ref. [42]) & $1215_{8}$ & $1214_{8}$ \\
Experiments (Ref. [52]) & \multicolumn{2}{c}{1188}
\end{tabular}

within the statistical fluctuations. Except the potential developed by Zhou et al., all the other EAM models used here show their inability of reproducing the experimental surface tension with deviations from experiments in the $27-65 \%$ range. We also note that all the EAM models underestimate the surface tension in line with recent simulations [4-6]. The EAM version from Sutton gives good results to reproduce shock properties and high pressure behavior [53], but gives unsatisfying results regarding surface tension calculation. In contrast, the performance of the EAM potential developed by Zhou et al. is excellent 

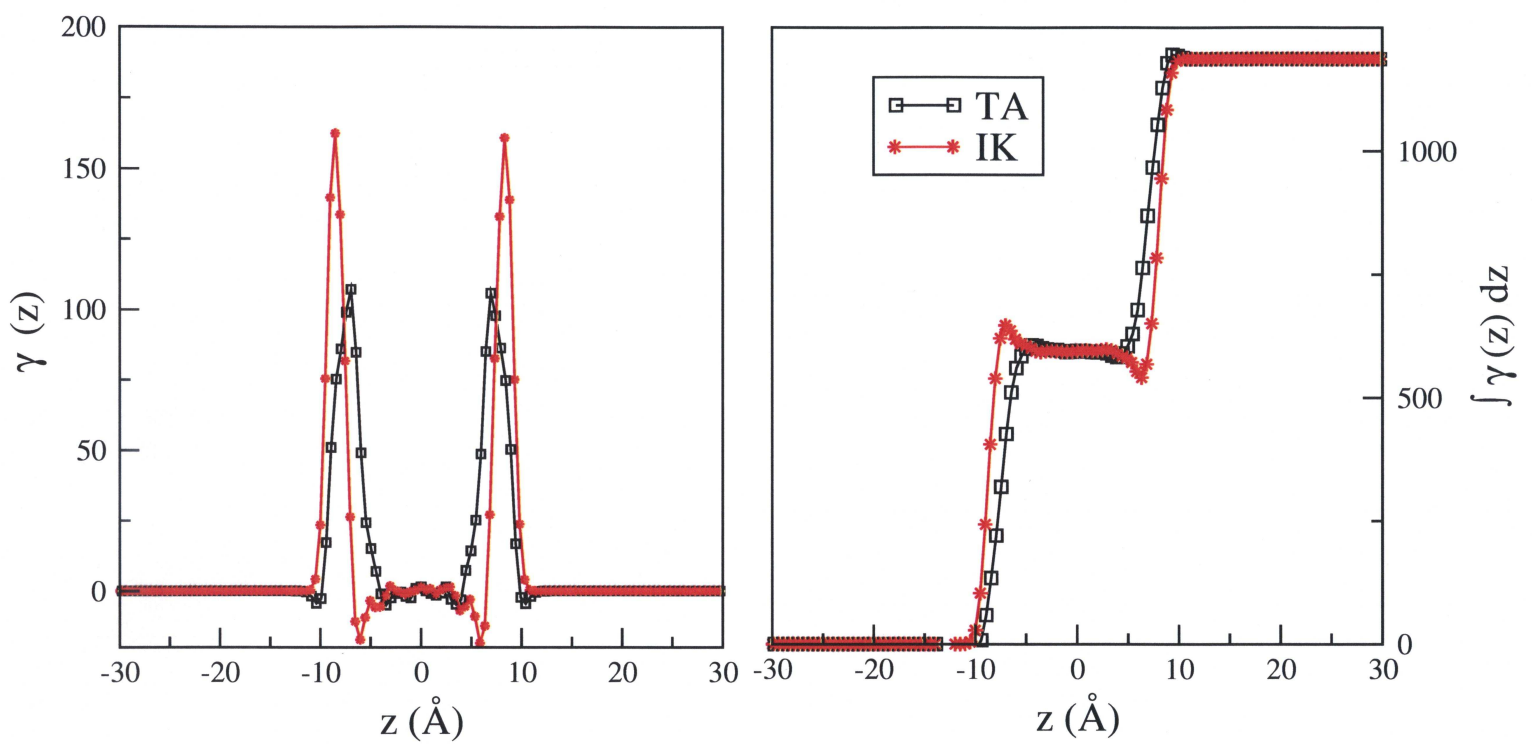

Fig. 3. Local element of the surface tension and its integration profile calculated using the Irving-Kirkwood (IK) and test area routes $(\mathrm{TA})$.

with a deviation of only $3 \%$ from experiments. From this comparison, we retain the EAM potential of Zhou et al. for investigating the temperature dependence of the surface tension of copper.

\subsection{Temperature dependence of the surface tension}

Before investigating the dependence of the surface tension of copper on the temperature, we focus on the temperature dependence of the molar volume using the EAM model developed by Zhou et al. [42]. NPT simulations of homogeneous copper at ambient pressure and several temperatures from 1000 to $2000 \mathrm{~K}$ were performed to determine the melting temperature of copper. The initial configurations were composed of a cubic centered lattice of 500 atoms in a cubic simulation box. We used periodic boundary conditions, and standard Monte Carlo moves (0.98 \% of translation moves and $0.02 \%$ of volume changes). Max-

imal amplitude of translations and volume changes were adjusted to get $40 \%$ of accepted moves at the end of the simulation.

Figure 4 shows the evolution of molar volume of copper as a function of temperature at $\mathrm{P}=1 \mathrm{~atm}$. The phase transition between solid and liquid states is located between 1400 and $1450 \mathrm{~K}$. The experimental melting temperature is equal to $1357 \mathrm{~K}$ at ambient pressure. Experimentally, the liquid density at the melting temperature and ambient pressure is 7.98 g.cm ${ }^{-3}$, whereas the simulations predict a liquid density of 7.69 g.cm ${ }^{-3}$ at $1450 \mathrm{~K}$, just above the melting point. The difference between experimental measures and calculation results with the EAM potential from Zhou is under $5 \%$. Nevertheless, we can note that the slope of the evolution of molar volume against temperature is greater in the solid phase (upper line in figure 


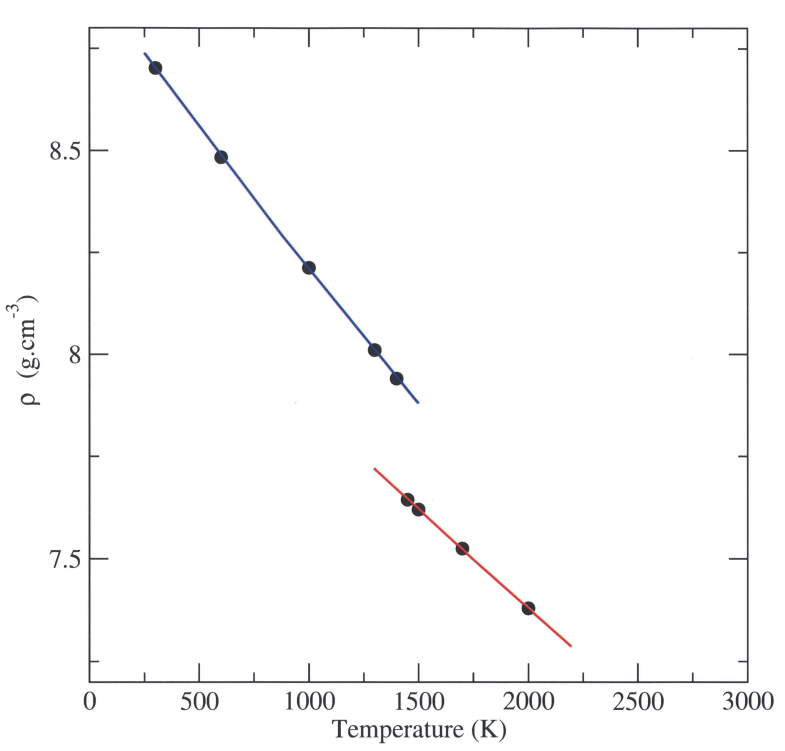

Fig. 4. Temperature dependence of the molar volume of copper. Symbols represent the simulation results, and lines are guides to the eye to show the slope of the evolution of molar volume against temperature.

4) than in the liquid phase (lower line in figure 4). This slope corresponds to the coefficient of thermal expansion, and it is supposed to be greater in the liquid than in the solid.

Figure 5 shows the temperature dependence of the surface tension of copper and Table 2 reports the different values of surface tensions calculated from IK, TA and TA2 routes at different temperatures. The experimental value is given for comparison. Very interestingly, Figure 5 establishes that the simulations using the EAM model developed by Zhou et al. give an excellent agreement with the experimental measurements (Harrison et al. [54], Naidich et al. [55]), and particularly with the more recent experimental data (Matsumoto et al. [52]). The maximum devi-

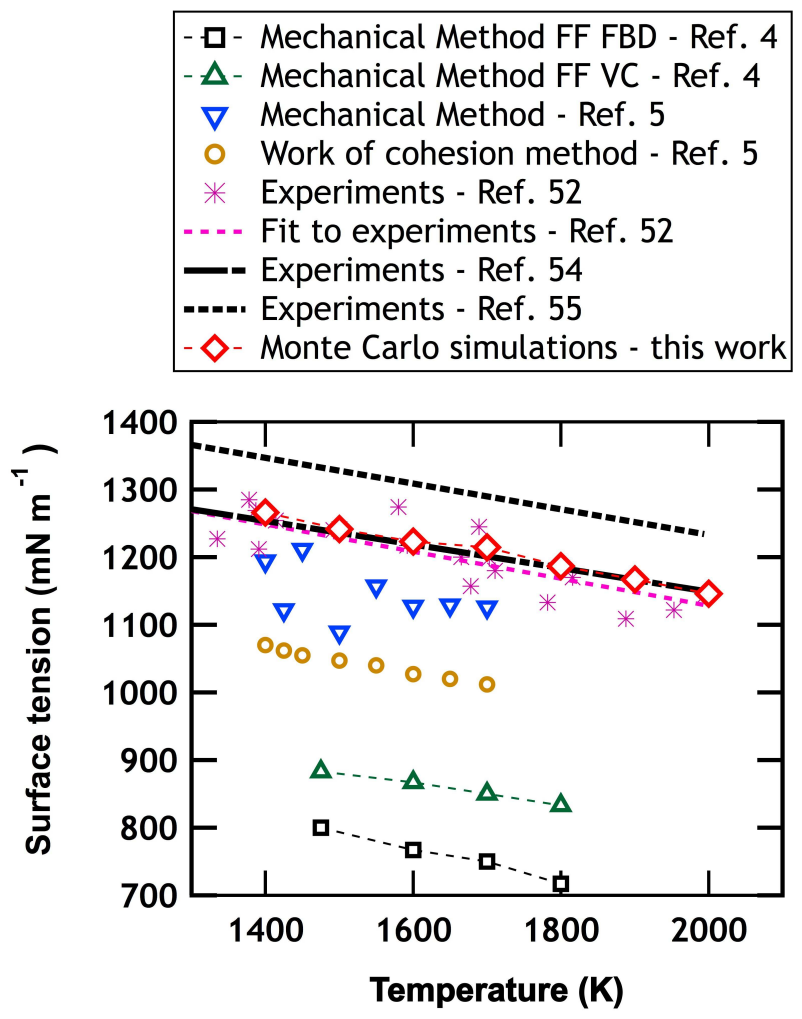

Fig. 5. Surface tension $\left(\mathrm{mN} \cdot \mathrm{m}^{-1}\right)$ of the liquid-vapor of copper calculated using the TA approach as a function of temperature.

We plot for comparison different results of surface tension from experiments, empirical predictions or molecular simulations as indicated in the legend.

ation from experiments is $26 \mathrm{mN} . \mathrm{m}^{-1}$ corresponding to a deviation of $2 \%$ from experiments. The quality of the prediction is excellent and better than those concerning the surface tension of organic compounds $[13,15,16,23,24,34$, 35]. Additionally, our calculations give better results in the prediction of the surface tension than previous works using the potentials (Webb III et al. [4], Hou et al. [5]) that underestimate this property. This success is probably due to the fact that this potential is well adapted to this type of calculation (it has been developed to simulate thin lay- 
Emeric Bourasseau et al.: Calculation of the surface tension of liquid copper from atomistic Monte Carlo simulations. 11

Table 2. Surface tension values $\left(\mathrm{mN}^{-1} \mathrm{~m}^{-1}\right)$ calculated using the TA, TA2 and IK methods. The experimental surface tensions are obtained by fitting the experimental data of Matsumoto et al. [52]. The resulting equation gives $\gamma(T)=$ $\left.1257+\gamma^{\prime}(T-1356)\right)$. The temperature coefficient defined by $\gamma^{\prime}=d \gamma(T) / d T$ is given for each method and for experiments.

\begin{tabular}{lcccc}
\hline $\mathrm{T}(\mathrm{K})$ & $\gamma_{T A}$ & $\gamma_{T A 2}$ & $\gamma_{I K}$ & $\gamma_{\text {exp. }}$ \\
\hline 1400 & $1264_{8}$ & $1265_{8}$ & $1266_{8}$ & 1248 \\
1500 & $1241_{11}$ & $1241_{11}$ & $1241_{11}$ & 1228 \\
1600 & $1222_{9}$ & $1222_{8}$ & $1223_{8}$ & 1208 \\
1700 & $1215_{8}$ & $1214_{8}$ & $1214_{8}$ & 1188 \\
1800 & $1186_{6}$ & $1186_{7}$ & $1187_{6}$ & 1168 \\
1900 & $1166_{6}$ & $1166_{6}$ & $1167_{6}$ & 1148 \\
2000 & $1146_{8}$ & $1145_{8}$ & $1146_{8}$ & 1128 \\
& & & & \\
& & & & \\
$\gamma^{\prime}\left(\mathrm{mN} . \mathrm{m}^{-1} . \mathrm{K}^{-1}\right)$ & -0.192 & -0.194 & -0.194 & -0.200 \\
\hline
\end{tabular}

ers of copper) and to the performance of the methodology used for the calculation of the surface tension.

The temperature coefficient $\gamma^{\prime}$, defined as $d \gamma(T) / d T$ in the equation $\gamma(T)=\gamma_{0}\left(T_{F}\right)+\gamma^{\prime}\left(T-T_{F}\right)$, where $T_{F}$ is the melting temperature, is of a great importance for liquid metals. This value is not well-known experimentally even for pure liquid metals. The prediction of this temperature coefficient remains quite challenging due to the scattering of the data coming from both theoretical and experimental works as shown in Figure 5. Linear fits of our results leads to slopes of $-0.192,-0.194,-0.194 \mathrm{mN} . \mathrm{m}^{-1} . \mathrm{K}^{-1}$ for the TA, TA2 and IK methods, respectively (see Table 2).
The range of experimental values $[52,54,55]$ for $\gamma^{\prime}$ is between -0.17 and $-0.21 \mathrm{mN} \cdot \mathrm{m}^{-1} \cdot \mathrm{K}^{-1}$. More recent measurements [52] give a slope of $-0.20 \mathrm{mN} \cdot \mathrm{m}^{-1} \cdot \mathrm{K}^{-1}$. The comparison with the more recent experimental value establishes a maximum deviation of $4 \%$ for the temperature coefficient. The large range of values of $\gamma^{\prime}$ and the scattering of the experimental surface tensions demonstrate the difficulty in measuring this property. A review of the experimental measurements can be found in Ref. [52]. The simulations show the performance of the EAM model developed by Zhou et al. to reproduce quantitatively the surface tension over a large range of temperatures and the temperature dependence of this property through the coefficient $\gamma^{\prime}$. This also shows the transferability of this EAM potential because the parameters of this potential were not developed from the surface tension.

\section{Conclusion}

Two-phase Monte Carlo simulations have been performed on the liquid-vapor interface of copper in order to reproduce the temperature dependence of the surface tension. The scattering of the experimental data and the small number of available simulated surface tension of liquid metal make the simulation of pure metal challenging from the choice of the potential and of the method.

In order to remove any dependence of the surface tension calculation on the methodology used, we have used the mechanical definition through the use of the IK method and the thermodynamic route by using the test area ap- 
proach. We have checked that the Monte Carlo simulations exhibit very-well converged surface tension values leading to an equivalence between the different definitions.

We have demonstrated that only the potential developed by Zhou et al. [42] allowed an excellent prediction of the surface tension of the liquid copper at a fixed temperature. We have used this potential to calculate the surface tension over a large range of temperatures. Interestingly, the agreement between the experimental values published in 2005 by Matsumoto et al. [52] and the simulated surface tensions is excellent with a maximum deviation from experiment of $2 \%$. The magnitude of this deviation is much smaller than that obtained in the prediction of the surface tension of liquid-vapor interface of organic compounds. Additionally, the simulated temperature coefficient of $0.194 \mathrm{mN} . \mathrm{m}^{-1} \cdot \mathrm{K}^{-1}$ matches very well the experimental value of $-0.20 \mathrm{mN} \cdot \mathrm{m}^{-1} \cdot \mathrm{K}^{-1}$ recently measured.

To conclude, atomistic simulations appear to be an interesting and powerful alternative to obtain surface tension of liquid metal, since the calculation results are less scattered that the experimental ones, and of course much easier to obtain. Nevertheless, the good accordance of the calculation results with the experimental measurements is mainly due to the potential, and what remains unclear in this work is why exactly this potential appears to be good to obtain the surface tension, and why other potentials do not. Additionally, the EAM potential developed by Zhou et al. [42] has been shown to be transferable on the surface tension whereas the parameters of this potential were not developed over this property. This work calls for further investigation concerning the choice of the input properties of the database for the development of a force field able to reproduce the surface tension.

\section{Acknowledgements}

All Monte Carlo simulations have been performed with the Gibbs code from IFP, CNRS and the Université Paris-Sud [56]. L. Soulard, from CEA, is acknowledged for fruitful discussions about the implementation of EAM potentials.

\section{References}

1. N. Eustathopoulos, E. Ricci, B. Drevet, Tech. Ing. M67, $1(1998)$

2. H.M. Lu, Q. Jiang, J. Phys. Chem. B 109, 15463 (2005)

3. N. Eustathopoulos, E. Ricci, B. Drevet, J. Cryst. Growth 191, $268(1998)$

4. E.B. WebbIII, G.S. Grest, Phys. Rev. Letters 86(2066), $2066(2001)$

5. H.Y. Hou, G.L. Chen, G. Chen, Y.L. Shao, Comput. Mater. Sci. 46(516-519), 516 (2009)

6. F. Calvo, J. Chem. Phys. 136, 154701 (2012)

7. P. Orea, J. Lopez-Lemus, J. Alejandre, J. Chem. Phys. 123, $114702(2005)$

8. M. Gonzalez-Melchor, P. Orea, J. Lopez-Lemus, F. Bresme, J. Alejandre, J. Chem. Phys. 122, 094503 $(2005)$

9. J.R. Errington, D.A. Kofke, J. Chem. Phys. 127, 174709 (2007) 
Emeric Bourasseau et al.: Calculation of the surface tension of liquid copper from atomistic Monte Carlo simulations. 13

10. F. Biscay, A. Ghoufi, F. Goujon, V. Lachet, P. Malfreyt, J. Chem. Phys. 130, 184710 (2009)

11. A. Trokhymchuk, J. Alejandre, J. Chem. Phys. 111, 8510 (1999)

12. J. Lopez-Lemus, J. Alejandre, Mol. Phys. 100, 2983 (2002)

13. F. Goujon, P. Malfreyt, A. Boutin, A.H. Fuchs, J. Chem. Phys. 116, 8106 (2002)

14. P. Grosfils, J.F. Lutsko, J. Chem. Phys. 130, 054703 (2009)

15. F. Goujon, P. Malfreyt, J.M. Simon, A. Boutin, B. Rousseau, A.H. Fuchs, J. Chem. Phys. 121, 12559 (2004)

16. C. Ibergay, A. Ghoufi, F. Goujon, P. Ungerer, A. Boutin, B. Rousseau, P. Malfreyt, Phys. Rev. E 75, 051602 (2007)

17. F. Goujon, C. Bonal, P. Malfreyt, Mol. Simulat. 35, 538 (2009)

18. G.J. Gloor, G. Jackson, F.J. Blas, E. de Miguel, J. Chem. Phys. 123, 134703 (2005)

19. C. Vega, E. de Miguel, J. Chem. Phys. 126, 154707 (2007)

20. A. Ghoufi, F. Goujon, V. Lachet, P. Malfreyt, Phys. Rev. E 77, $031601(2008)$

21. M. Guo, B.C.Y. Lu, J. Chem. Phys. 106, 3688 (1997)

22. V.K. Shen, R.D. Mountain, J.R. Errington, J. Phys. Chem. B 111, 6198 (2007)

23. F. Goujon, P. Malfreyt, A. Boutin, A.H. Fuchs, Mol. Simulat. 27, 99 (2001)

24. F. Biscay, A. Ghoufi, F. Goujon, V. Lachet, P. Malfreyt, J. Phys. Chem. B 112, 13885 (2008)

25. J. Janecek, H. Krienke, G. Schmeer, Condens. Matter Phys. 10, 415 (2007)

26. C. Nieto-Draghi, P. Bonnaud, P. Ungerer, J. Phys. Chem. C 111, 15686 (2007)
27. F. Biscay, A. Ghoufi, V. Lachet, P. Malfreyt, Phys. Chem. Chem. Phys. 111, 6132 (2009)

28. N. Ferrando, V. Lachet, J. Pérez-Pellitero, A.D. Mackie, P. Malfreyt, A. Boutin, J. Phys. Chem. B 115, 10654 (2011)

29. A. Ghoufi, F. Goujon, V. Lachet, P. Malfreyt, J. Chem. Phys. 128, 154716 (2008)

30. A. Ghoufi, P. Malfreyt, Phys. Rev. E 83, 051601 (2011)

31. A. Ghoufi, F. Goujon, V. Lachet, P. Malfreyt, J. Chem. Phys. 128, 154718 (2008)

32. J. Alejandre, D.J. Tildesley, G.A. Chapela, J. Chem. Phys. 102, $4574(1995)$

33. J.C. Neyt, A. Wender, V. Lachet, P. Malfreyt, J. Phys. Chem. B 115, 9421 (2011)

34. F. Biscay, A. Ghoufi, P. Malfreyt, J. Chem. Phys. 134, 044709 (2011)

35. F. Biscay, A. Ghoufi, V. Lachet, P. Malfreyt, J. Phys. Chem. C 115(0), 8670 (2011)

36. F. Biscay, A. Ghoufi, V. Lachet, P. Malfreyt, J. Chem. Phys. 131, 124707 (2009)

37. F. Biscay, A. Ghoufi, V. Lachet, P. Malfreyt, J. Phys. Chem. B 113, 14277 (2009)

38. J.C. Neyt, A. Wender, V. Lachet, P. Malfreyt, J. Phys. Chem. C 116, 10563 (2012)

39. M.S. Daw, M.I. Baskes, Phys. Rev. B 29, 6443 (1984)

40. P.M. Agrawal, B.M. Rice, D.L. Thompson, Surf. Science 515(21-35), 21 (2002)

41. J.O. Hirschfelder, R.B. Bird, E.L. Spotz, J. Chem. Phys. 16(968-981), 968 (1948)

42. X.W. Zhou, R.A. Johnson, H.N.G. Wadley, Phys. Rev. B 69(144113), 144113 (2004) 
14 Emeric Bourasseau et al.: Calculation of the surface tension of liquid copper from atomistic Monte Carlo simulations.

43. A.B. Belonoshko, R. Ahuja, O. Eriksson, B. Johansson, Phys. Rev. B 61(3838-3844), 3838 (2000)

44. F. Cleri, V. Rosato, Phys. Rev. B 48, 22 (1993)

45. A.P. Sutton, J. Chen, Mag. Lett. 61(139), 139 (1990)

46. J.S. Rowlinson, B. Widom, Molecular Theory of Capillarity (Clarendon Press, Oxford, 1982)

47. J.G. Kirkwood, F.P. Buff, J. Chem. Phys. 17, 338 (1949)

48. J.H. Irving, J.G. Kirkwood, J. Chem. Phys. 18, 817 (1950)

49. J.P.R.B. Walton, D.J. Tildesley, J.S. Rowlinson, J.R. Henderson, Mol. Phys. 48, 1357 (1983)

50. J.P.R.B. Walton, K.E. Gubbins, Mol. Phys. 55, 679 (1985)

51. A. Ghoufi, P. Malfreyt, J. Chem. Phys. 136(024104), $024104(2012)$

52. T. Matsumoto, H. Fujii, T. Ueda, M. Kamai, K. Nogi, Meas. Sci. Technol. 16, 432 (2005)

53. O. Durand, L. Soulard, Journal of App. Phys. 111(044091), 044091 (2012)

54. Harrison, J. Chem. Phys. 9(12), 1111 (1977)

55. Y.V. Naidich, V.N. Eremenko, V.V. Fesenko, M.I. Vasiliu, L.F. Kirichenko, The role of surface phenomena in metallurgy (Ed. V. N. Eremenko, 1963)

56. P. Ungerer, B. Tavitian, A. Boutin, Applications of Molecular Simulation in the Oil and Gas Industry (IFP Publications, 2005) 